\title{
Transport Catastrophe Analysis as an Alternative to a Monofractal Description: Theory and Application to Financial Crisis Time Series
}

\author{
Sergey A. Kamenshchikov ${ }^{1,2}$ \\ ${ }^{1}$ Faculty of Physics, Moscow State University of M. V. Lomonosov, Leninskie Gory, Moscow 119991, Russia \\ ${ }^{2}$ Analytics Division, IFC Markets Corp., 145-157 St. John Street, London EC1V 4PY, UK
}

Correspondence should be addressed to Sergey A. Kamenshchikov; kamphys@gmail.com

Received 25 May 2014; Revised 3 August 2014; Accepted 18 August 2014; Published 14 September 2014

Academic Editor: Norbert Marwan

Copyright (c) 2014 Sergey A. Kamenshchikov. This is an open access article distributed under the Creative Commons Attribution License, which permits unrestricted use, distribution, and reproduction in any medium, provided the original work is properly cited.

\begin{abstract}
The goal of this investigation was to overcome limitations of a persistency analysis, introduced by Benoit Mandelbrot for monofractal Brownian processes: nondifferentiability, Brownian nature of process, and a linear memory measure. We have extended a sense of a Hurst factor by consideration of a phase diffusion power law. It was shown that precatastrophic stabilization as an indicator of bifurcation leads to a new minimum of momentary phase diffusion, while bifurcation causes an increase of the momentary transport. An efficiency of a diffusive analysis has been experimentally compared to the Reynolds stability model application. An extended Reynolds parameter has been introduced as an indicator of phase transition. A combination of diffusive and Reynolds analyses has been applied for a description of a time series of Dow Jones Industrial weekly prices for the world financial crisis of 2007-2009. Diffusive and Reynolds parameters showed extreme values in October 2008 when a mortgage crisis was fixed. A combined R/D description allowed distinguishing of market evolution short-memory and long-memory shifts. It was stated that a systematic large scale failure of a financial system has begun in October 2008 and started fading in February 2009.
\end{abstract}

\section{Introduction}

In 1955, the American researcher Hassler Whitney has created a mathematical foundation of a modern catastrophe theory-the theory of mapping singularities [1]. It includes investigations of peculiarity classes that appear for mapping of one two-dimensional surface to another one. Whitney has found out two stable types of mappings - types that have not been destroyed after negligible deformations of surfaces or their projections. These types of mappings have been generalized for arbitrary manifolds with dimensions up to 10 by Whitney's followers; see, for example, [2]. One of them led to the discrete change of a system's characteristic state"cusp" catastrophe. It is represented for a one-dimensional case in Figure 1. The multiplicity or uncertainty is maximal in the unstable area of C-vicinity. According to [1], the disruption $D \rightarrow E$ appears as a fusion of stable and unstable regimes, marked by ovals. In terms of a bifurcation theory, this one-dimensional evolution corresponds to the saddlenode fusion in a phase space. Another type of destabilization is a self-oscillating destabilization, suggested by $\mathrm{H}$. Poincare (1879) in his dissertation thesis. It has been proved by A. Andronov and E. Leontovich in 1939.

According to the Andronov-Leontovich theorem [3], a birth of a new limited cycle in a phase space is realized by a transition through a stable equilibrium zone; that is, the system should return to the stabilization before a new bifurcation occurs. A birth of a new cycle is preceded by the distortion and death of a previous quasistable regime.

A "calm before storm" or effect of small scale oscillations suppression before bifurcation has been noticed by Dubovikov et al. in [4] as well. They have studied a behavior of financial time series by the use of Hurst monofractal parameter of stability. Anatoly Neishtadt has shown that a delay of the dynamic bifurcation exists in case of all known analytical nonlinear systems [5] for adiabatic change 


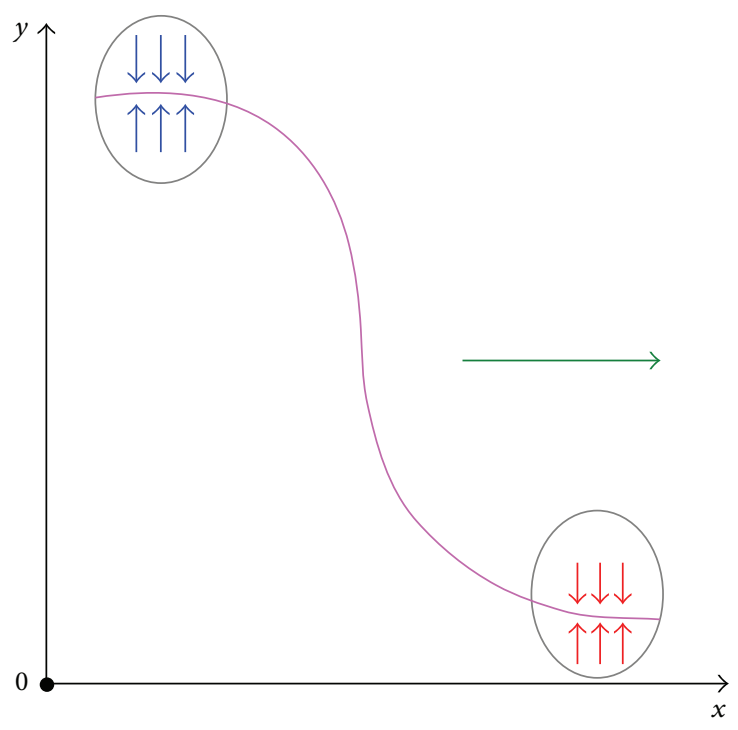

(a)

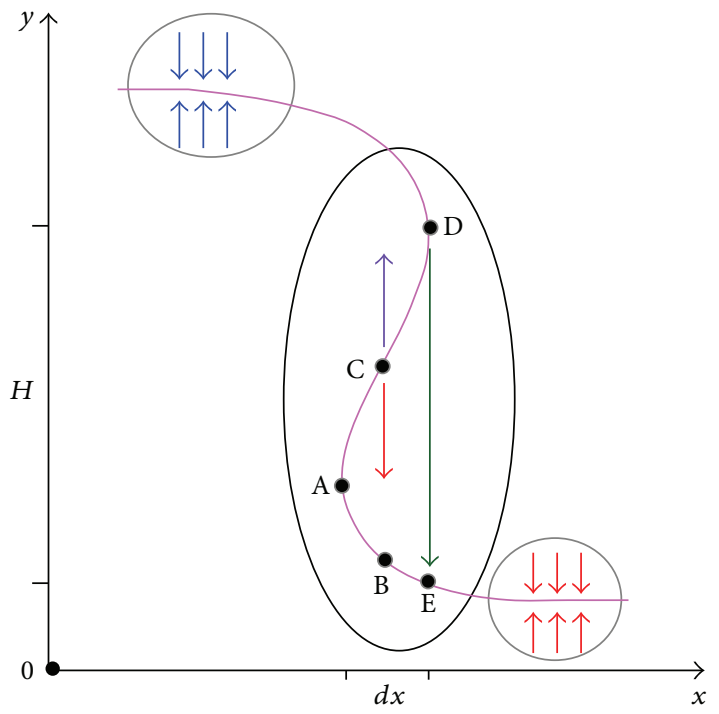

(b)

FIGURE 1: Appearance of Whitney's "cusp."

of control parameter. It means that inertial properties resist a new synchronization-the system needs time for the restructuring as it happens in case of Ising model of magnetic domains. A delay depends on the clusters interaction and an intensity of the external "field," that is, macroscale influence. A comfortable choice of a macroscopic control parameter has been suggested in [6] on basis of Reynolds parameter for turbulent streams:

$$
R(t)=f(\vec{\Pi}(t))=\frac{q^{+}(t)}{q^{-}(t)} .
$$

Here, $R(t)$ is the basic phase parameter and $\vec{\Pi}(t)$ is the set of microscopic control parameters-parametric vector. Quantities $q^{+}$and $q^{-}$correspond to power input and output per system unit. In given description, bifurcation corresponds to the transition of an equilibrium state $R=1$ :

$$
\begin{aligned}
R\left(t_{0}\right) & =1 \Longrightarrow \uparrow q^{+}(t) \Longrightarrow \uparrow R(t) \Longrightarrow R(t)>1 \\
& \Longrightarrow \uparrow q^{-}(t) \Longrightarrow \uparrow R(t) \Longrightarrow R\left(t_{1}\right)=1, \\
R\left(t_{0}\right) & =1 \Longrightarrow \downarrow q^{+}(t) \Longrightarrow \downarrow R(t) \Longrightarrow R(t) \prec 1 \\
& \Longrightarrow \downarrow q^{-}(t) \Longrightarrow \uparrow R(t) \Longrightarrow R\left(t_{1}\right)=1 .
\end{aligned}
$$

Here, $\uparrow$ and $\downarrow$ show a finite increase and decrease of corresponding parameter for $t_{1}>t>t_{0}$. The delay between a new cycle appearance and macroscale excitation is defined by the inertial properties of system domains. A new bifurcation has to pass through an equilibrium quasistable state of $R=1$.

\section{Monofractal Analysis as an Indicator of Stability and Its Limitations}

Inability to define strictly a set of control parameters and a global excitation balance obliged researchers to look for statistical measures of the system stability. One of the classical approaches is a monofractal analysis, suggested by Benoit Mandelbrot.

According to Mandelbrot and his revolutionary work [7], scale invariance is the necessary property of fractals.

However, we should note that chaotic natural systems have scale characteristic limits. For example, a turbulent flow has an internal microscale, defined by inertial viscous forces, and an external macroscale, defined by external hydrodynamic influence. Such type of system was denoted as quasifractal by Mandelbrot, because it has a satisfactory fractal description only within given scale limits $\varepsilon_{0} \prec$ $\varepsilon \prec E$. An application of a fractal description to the time series $f\left(t_{i}\right)$ meant an investigation of statistical properties in case of several time resolutions $\varepsilon={ }_{\Delta} t$.

A single characteristic in frame of a classical monofractal description, introduced by Mandelbrot, is a Hurst factor. It was induced through the relation of fractional Brownian motion (FBM) [7]. An idea of FBM introduction was the inability to explain deviations from normal distributions in some natural systems, for example, financial markets. ParetoLevy distributions have been obtained as particular cases of such abnormal behavior.

Let us consider a standard Brownian motion (SBM) time series $B(t)$, which satisfies a normal distribution. Then, an FBM increment can be expressed in the following way:

$$
\begin{aligned}
B_{H}\left(t_{2}\right)-B_{H}\left(t_{1}\right)= & \frac{1}{\Gamma(H+1 / 2)} \int_{-\infty}^{t_{2}}\left(s-t_{2}\right)^{1 / 2-H} d B(s) \\
& -\frac{1}{\Gamma(H+1 / 2)} \int_{-\infty}^{t_{1}}\left(s-t_{1}\right)^{1 / 2-H} d B(s) .
\end{aligned}
$$

A given increment is expressed through the fractal derivatives of SBM with a factor $k=(0.5-H), 0 \prec H \prec 1$. This factor 
defines a deviation from a standard Markovian Brownian motion $(H=0.5)$. FBH allows obtaining anomalous distributions with "thick tails" and a flexible explaining of flights. According to [8], an expectation of FBM deviation is self-affine:

$$
\begin{gathered}
E\left(\left[B_{H}(t+T)-B_{H}(t)\right]^{2}\right)=V_{H} \cdot T^{2 H}, \\
V_{H}=\left(\frac{1}{\Gamma(H+1 / 2)}\right)^{2} \\
\cdot\left[\int_{-\infty}^{0}\left[(1-s)^{H-1 / 2}-(-s)^{H-1 / 2}\right]^{2} d s+\frac{1}{2 H}\right] .
\end{gathered}
$$

This means that probable amplitude of the deviation depends on a time scale and a Hurst factor $H$ of system's memory. If $H=0.5$, then relation (4a) corresponds to Einstein's law of Brownian walks:

$$
E\left([B(t+T)-B(t)]^{2}\right)=V_{0.5} \cdot T .
$$

If $H \neq 0.5$, we achieve an anomalous transport that includes Levy flights and "thick tails" of distribution for $H>0.5$. Despite the charm of this approach, it has several limitations, enumerated below.

(a) FBM Is Achieved as Weighted Averaged Brownian Motion. According to the original work of Mandelbrot and van Ness [7], "FBM of the exponent $H$ is a moving average of $d B(t)$ in which past increments of $B(t)$ are weighted by the kernel $(t-s)^{H-1 / 2}$." The weights are defined on the basis of time distance between current moment and previous states $\Delta=$ $\left(s-t_{j}\right)$. The intensity of a history influence is determined by a memory factor $0 \prec H \prec 1$. However, FBM operator assumes SBM kernel for weighted average. It means that FBM is considered as dynamical moving weighted integration of standard Brownian process.

If we calibrate FBM such that $B_{H}\left(t_{0}\right)=0$, then an absolute value can be expressed in the following way:

$$
{ }_{\Delta} B_{H}(t)=\frac{1}{\Gamma(H+1 / 2)} \int_{-\infty}^{t}(s-t)^{1 / 2-H} \cdot d B(s) .
$$

If we consider a motion only in negligible time range $(t-d s, t)$, then this relation can be simplified:

$$
\begin{aligned}
d B_{H}(t) & =\frac{1}{\Gamma(H+1 / 2)} d s^{1 / 2-H} \cdot B^{\prime}(s) \cdot d s \\
& =\frac{d s^{1 / 2-H}}{\Gamma(H+1 / 2)} \cdot d B .
\end{aligned}
$$

Up to a constant factor, this relation corresponds to the SBM increment. In such a way, FBM assumes limitations of Markovian process that should be satisfied for small time deviations.

(b) An Increment of the FBM Has an Infinite Exact Energy. As it was stated by Mandelbrot and van Ness [7], a first fractal derivative of FBM and consequently its energy diverges for the range $0 \prec H \prec 1$. To overcome this obstacle, they have introduced a smoothed derivative where a range of smoothing $\delta$ is defined artificially:

$$
\begin{gathered}
B_{H}^{\prime}(t, \delta)=-\int_{-\infty}^{\infty} B_{H}(s) d \varphi(t-s), \\
\varphi(t)=\frac{1}{\delta}, \quad t \in[0, \delta], \\
\varphi(t)=0, \quad t \prec 0, \\
\varphi(t)=0, \quad t>\delta .
\end{gathered}
$$

However, that is not the only procedure to introduce "physical" derivative (we may use a weighted derivative as well) and that is why the universality of a dynamic description is lost.

(c) Hurst Factor Expresses a Linear Measure of Memory and Is Not Applicable for Nonlinear Cases. This remark needs a certain clarification. According to [7], a linear autocorrelation function of a first derivative can be expressed in the following way:

$$
\begin{aligned}
C_{H}(\tau, \delta) & =E\left(\frac{\partial B_{H}}{\partial t}(t, \delta) \mid \frac{\partial B_{H}}{\partial t}(t+\tau, \delta)\right) \\
& =V_{H} \cdot H \cdot(2 H-1)|\tau|^{2(H-1 / 2)} .
\end{aligned}
$$

It is a quadratic function of Hurst factor and again depends on the artificial smoothing parameter $\delta$. Cases of $H>$ 0.5 and $H \prec 0.5$ correspond to the persistent and antipersistent trends correspondingly [4, 8]. In case of Markovian SBM, $H=0.5$ and $C_{H}(\tau, \delta)=0$. For Markovian process, a probability connection is stated by Chapman-Kolmogorov equation which is not linear in general:

$$
\begin{aligned}
W & \left(x_{3}, t_{3} \mid x_{1}, t_{1}\right) \\
& =\int d x_{2} W\left(x_{3}, t_{3} \mid x_{2}, t_{2}\right) \cdot W\left(x_{2}, t_{2} \mid x_{1}, t_{1}\right) .
\end{aligned}
$$

In fact, a Hurst factor as a memory measure can be applied for the characterization of linear trends in regard to the function ${ }_{\Delta} B_{H}(t)$, but according to a standard FBM model it cannot be used generally for the indication of precatastrophic stabilization, considered in Section 1.

(d) Hurst Factor Is a Single Macroscopic Fractal Characteristic. It means that a detailed structure of a nonuniform phase area should be investigated with use of additional parameters. If self-affine properties depend on space-time scale, then other models can be used for a more subtle description-for example, the multifractal detrended fluctuation analysis [9].

Limitations, mentioned above, can be partially overcome by the introduction of extended Hurst analysis.

\section{Extended Interpretation of Hurst Parameter}

In this section, we shall consider a diffusive approach to the characterization of precatastrophic stabilization effect (PS-effect), noted in Section 1. This description assumes an introduction of a second transport factor, used in a standard Fokker-Planck equation (11). 
This equation has been derived on a basis of ChapmanKolmogorov equation (10) and, thus, is applied for Markovian processes:

$$
\begin{array}{r}
\frac{\partial P(x, t)}{\partial t}=\frac{1}{2} \cdot \frac{\partial}{\partial x}\left(D(x) \cdot \frac{\partial P(x, t)}{\partial x}\right), \\
D(x)=\lim _{\Delta} t \rightarrow 0\left(\frac{\left\langle\left\langle{ }_{\Delta} x^{2}\right\rangle\right\rangle}{{ }_{\Delta} t}\right)=\frac{\left\langle\left\langle{ }_{\Delta} x^{2}\right\rangle\right\rangle}{{ }_{\Delta} t_{\min }} .
\end{array}
$$

Here, double brackets designate an averaging of an initial coordinate:

$$
b\left(x,{ }_{\Delta} t\right)=\int\left(x-x_{0}\right)^{2} \cdot W\left(x, x_{0},{ }_{\Delta} t\right) d x_{0}=\left\langle\left\langle{ }_{\Delta} x^{2}\right\rangle\right\rangle .
$$

It has been shown in [9] that systems of phase mixing have a second transport factor $D$ with explicit time dependence, expressed through the specific energy of characteristic vector:

$$
\begin{aligned}
& D(x, t)=\frac{\left\langle\left\langle{ }_{\Delta} x^{2}\right\rangle\right\rangle}{{ }_{\Delta} t_{\min }}={ }_{\Delta} t_{\min } \cdot \int \varepsilon(x, t) \cdot W\left(x, x_{0},{ }_{\Delta} t\right) d x_{0} \\
&={ }_{\Delta} t_{\min } \cdot\langle\langle\varepsilon(x, t)\rangle\rangle \\
& \varepsilon(x, t)=\lim _{\Delta} t \rightarrow 0 \\
& \rightarrow
\end{aligned}
$$

Let us introduce a variable time lag $T=t-t_{0}$ and a power regression of the transport factor that will be denoted as a dynamic diffusion below:

$$
D\left(t_{0}, T\right)=D_{0}\left(t_{0}\right) \cdot T^{\kappa} .
$$

Then, an expectation of the stochastic shift can be represented in the following way:

$$
E\left(\left[x\left(t_{0}+T\right)-x\left(t_{0}\right)\right]^{2}\right)=D_{0}\left(t_{0}\right) \cdot T^{\kappa+1} .
$$

A comparison of this relation with (4a) allows expressing the Hurst factor through a stability coefficient $\kappa: H=(\kappa+$ $1) / 2$. Unlike an FBM procedure, we made no assumptions regarding a microscale probability distribution function. That is why a generalized Hurst parameter has no obligatory preliminary limitations in frame of this model $-\infty \prec H \prec$ $\infty$. It does not create an artificial energy divergence as well and may be applied to natural systems directly without smoothing. However, an extended Hurst analysis still has a limitation of Markovian processes (12). A critical value of $H_{\mathrm{cr}}=0.5$ corresponds to the constant diffusion case $\kappa=$ 0 and is a boundary of the diffusive expansion $H>0.5$ and the diffusive contraction $H \prec 0.5$ of a characteristic area. Thus, a generalized Hurst factor is a measure of the attractor stability. We may introduce a potential of attraction on the basis of a diffusive scale:

$$
\Lambda_{D}=\sqrt{\left\langle\left\langle{ }_{\Delta} x^{2}\right\rangle\right\rangle}=\sqrt{D_{0}\left(t_{0}\right)} \cdot T^{H}
$$

Then, a diffusive acceleration can be represented in the following way:

$$
\frac{\partial^{2} \Lambda}{\partial T^{2}}=\sqrt{D_{0}\left(t_{0}\right)} H(H-1) \cdot T^{H-2}=-\frac{\partial V_{\mathrm{eff}}}{\partial x} .
$$

Here, $V_{\text {eff }}$ is an efficient volume potential of attraction of a given phase space. In regard to the time series analysis, it characterizes a volatility of a considered time series:

$$
\int \sqrt{D_{0}\left(t_{0}\right)} H(1-H) \cdot T^{H-2} d x=V_{\mathrm{eff}}
$$

It is an integral characteristic of a manifold internal interaction. According to relation (19), a system may lose memory even for "persistent" case of $H>0.5$.

A sense of a generalized Hurst factor can be clarified with a use of spectral description. If we introduce a characteristic frequency $_{\Delta} t=1 / f$, then according to the relation (17) the following formula may be represented for a dynamic diffusion spectrum:

$$
D\left(t_{0}, f\right)=D_{0}\left(t_{0}\right) \cdot f^{-\kappa} .
$$

We may note that the case of $H>H_{\text {cr }}$ corresponds to the large scale transport, while if $H \prec H_{c r}$, a microscale energy absorption is more intense. A shift of a basic absorption band from microscale range to macroscale range corresponds to catastrophic behavior, when a coherent motion and resonances appear.

It should be remarked that, in general case of a nonuniform phase area, the $\kappa$ factor may in its turn depend on the frequency. An additional nonlinear form of the diffusive law then may be introduced. However, relation (20) can still be used as the initial approach for the description of macroscopic diffusive properties.

\section{Diffusive Analysis and $R$-Bifurcations}

In Section 1, we have considered a precatastrophic stabilization effect (PS-effect) as a first necessary condition of the bifurcation. It should be marked that a PS-effect leads to the small scale spectrum band intensification with a following transition to a large scale transport. One of the possible ways that can be used to compare macro-/microtransport properties is represented by

$$
I\left(t_{0}\right)=\frac{\int_{T_{\min }}^{T_{\max } / 2} D\left(t_{0}, T\right) d T}{\int_{T_{\min }}^{T_{\max } / 2} D\left(t_{0}, T\right) d T} .
$$

Here, an integral stabilization factor $I$ is expressed through the relation of small frequency and high frequency integrals. A total integration range $\left(T_{\min }, T_{\max }\right)$ is defined with an account of measurement resolution.

Another alternative is a momentary transport analysis of the uniform Markovian time series $x_{i}=x\left(t_{i}\right)$. It can be outlined on a basis of expression (13). Factor (22) allows defining an average transport for the period $T=t_{i}-$ $t_{i-N}$ and reaches a new minimum during a precatastrophic 
stabilization phase: ${ }_{\Delta} D\left(x_{i}, T\right)=0$. A disruption leads to an increase of the momentary transport due to a large scale motion:

$$
\begin{gathered}
D\left(x_{i}, T\right)=\frac{\left\langle\left\langle{ }_{\Delta} x_{i}^{2}\right\rangle\right\rangle}{t_{i}-t_{i-N}}=\frac{\sum_{j=i-N}^{i}\left(x_{i}-x_{j}\right)^{2}}{T}, \\
{ }_{\Delta} D\left(x_{i}, T\right)=0 \Longrightarrow{ }_{\Delta} D\left(x_{i}, T\right)>0 .
\end{gathered}
$$

An alternative choice of a control parameter has been suggested in Section 1 on the basis of an extended Reynolds factor. It may be denoted as a basic phase parameter $R(t)$. According to mechanisms (2), a bifurcation corresponds to the state $R(t) \neq 1$ and a following formation of a new quasicycle such that $R(t) \rightarrow 1$. As it was shown in [10], two principle types of disruption are possible. Let us use a dynamic entropy of Kolmogorov $h_{d}[11]: h_{d}=\langle h(\vec{x}(t))\rangle$.

Here, averaging in phase space is designated as \langle\rangle and averaged quantity can be expressed as sum of positive Lyapunov factors $h_{i}^{+}$for each dimension of generalized phase space:

$$
\begin{gathered}
h=\sum_{i=N}^{K} h_{i}^{+}=\ln \left(\prod_{i=N}^{K} \sigma_{i}^{+}\right), \\
\sigma_{i}^{+}(t)=\frac{\left|\delta x_{i}(t)\right|}{\left|\delta x_{i}^{0}\right|} .
\end{gathered}
$$

We may denote $\vec{x}(t)$ as characteristic phase vector of system state. Factor $\sigma_{i}^{+}$shows distance growth $\delta x_{i}(t)$ in $i$ direction for two infinitely closely located points in phase space. Condition of stationary state then is equal to $h=0$ or $\sigma_{i}^{+}=$ $1(i=\overline{N, K})$.

Let us introduce a relation for specific system power:

$$
q(t)=\frac{\delta}{\delta t}\left(\sum_{i=1}^{K} \frac{v_{i}^{2}}{2}\right)=\sum_{i=1}^{K} v_{i} \cdot \dot{v}_{i}=q^{+}(t)-q^{-}(t) .
$$

System stability condition leads to $h_{i} \leq 0$ and $\sigma_{i} \leq 1$. Given inequalities lead to expression (25) for velocity components $v_{i}(i=\overline{1, K})$ :

$$
\frac{\left|\delta x_{i}(t)\right|}{\left|\delta x_{i}^{0}\right|}=\frac{\left|\delta x_{i}(t) / \delta t\right|}{\left|\delta x_{i}^{0} / \delta t\right|}=\frac{\left|v_{i}(t)\right|}{\left|v_{i}^{0}\right|}=\alpha(t) \quad 0 \prec \alpha(t) \leq 1 .
$$

Relation (25) in fact allows receiving components of acceleration $\dot{v}_{i}(t)$ :

$$
\begin{aligned}
& \lim _{\Delta} t \rightarrow 0\left(\frac{\left|v_{i}(t)\right|-\left|v_{i}^{0}\right|}{{ }_{\Delta} t}\right) \leq 0, \\
& \dot{v}_{i}(t)=\lim _{\Delta} t \rightarrow 0 \\
& { }_{\Delta} t
\end{aligned}
$$

In such a way, a consideration of specific power $q(t)$ can be reduced to two cases: (a) $\delta x_{i}(t)>0$ and $\left|\delta x_{i}(t)\right|=$ $\delta x_{i}(t)$; (b) $\delta x_{i}(t) \leq 0$ and $\left|\delta x_{i}(t)\right|=-\delta x_{i}(t)$. Signs of $\delta x_{i}(t)$ and $\delta x_{i}^{0}$ match-this condition is obligatory for definition of Lyapunov factors. Then, $\alpha(t)$ does not depend on initial sign of coordinate shift $\delta x_{i}^{0}$.

For cases (a) and (b), we then receive (a) $\dot{v}_{i} \prec 0$ and $v_{i} \succ$ 0 ; (b) $\dot{v}_{i} \geq 0$ and $v_{i} \leq 0$. In both cases with use of relation (25) we receive that $q(t) \leq 0$. According to the definition of a basic phase parameter, this means that $R(t) \leq 1$ for $h_{d} \leq$ 0 . Condition of $R_{f}$, that is, $R(t)=1$, corresponds to a new stable regime. Thus, use of $R(t)$ as control parameter must be delimited for two types of system [6]: (a) accelerator, $\dot{v}_{i}(t)>$ 0 ; (b) decelerator, $\dot{v}_{i}(t) \leq 0$. For first type of system motion stability loss and bifurcation are realized for $R(t) \prec 1$, while decelerator comes to transition only for $R(t)>1$. In both cases, a transition corresponds to the following requirement:

$$
\begin{gathered}
R(t)=1 \Longrightarrow R(t) \neq 1 \\
{ }_{\Delta} \varepsilon(t)=0 \Longrightarrow{ }_{\Delta} \varepsilon(t) \neq 0 .
\end{gathered}
$$

\section{Reynolds and Diffusive Analysis: Application to Financial Markets}

In this section we shall consider several illustrations of diffusive and $R$-analysis applications to the financial time series. Let us consider a time period of 16.07.2006-21.02.2010 which includes two significant phases-a beginning of the world financial crisis and a gradual recovering. According to the analysis of Soros [12], a preliminary origin of the crisis corresponds to the falling of bank liquidity in August 2007. In September 2008, it caused a failure of greatest American mortgage agencies: Lehman Brothers, Fannie Mae, and Freddie Mac.

Only in January 2009 has US Federal Reserve started the fourth supporting program of financial stabilization (QE4) that led to a preliminary recovering of a financial system.

Let us analyze a time series of DJI weekly prices-an index price was defined at the trading end of each Friday-the end of a trading week. In Figure 2 it is shown that an upward trend, marked as rising corridor, has been broken in August 2007.

A total collapse of this index corresponds to the failure of mortgage agencies in October 2008. Let us demonstrate an application of two approaches that were considered aboveanalysis of a basic phase parameter $R(t), R$-analysis, and a diffusive analysis, $D$-analysis.

Both will be applied for the discovering of new system disruptions, corresponding to macroscale shifts of price:

$$
\begin{gathered}
\varepsilon_{i}=\frac{1}{2}\left(\frac{x_{i}-x_{i-1}}{{ }_{\Delta} t_{i}}\right)^{2}, \quad{ }_{\Delta} \varepsilon_{i}=\varepsilon_{i}-\varepsilon_{i-1}, \\
{ }_{\Delta} t_{i}={ }_{\Delta} t=\text { const }, \quad i=\overline{1, N} .
\end{gathered}
$$

The set of relations (28) expresses a difference approximation of the parameter ${ }_{\Delta} \varepsilon_{i}\left(t_{i}\right)$. According to the conditions, considered above, an attraction sign should be defined simultaneously: $\dot{v}_{i}(t)>0$ or $\dot{v}_{i}(t) \prec 0$ scenario must be 


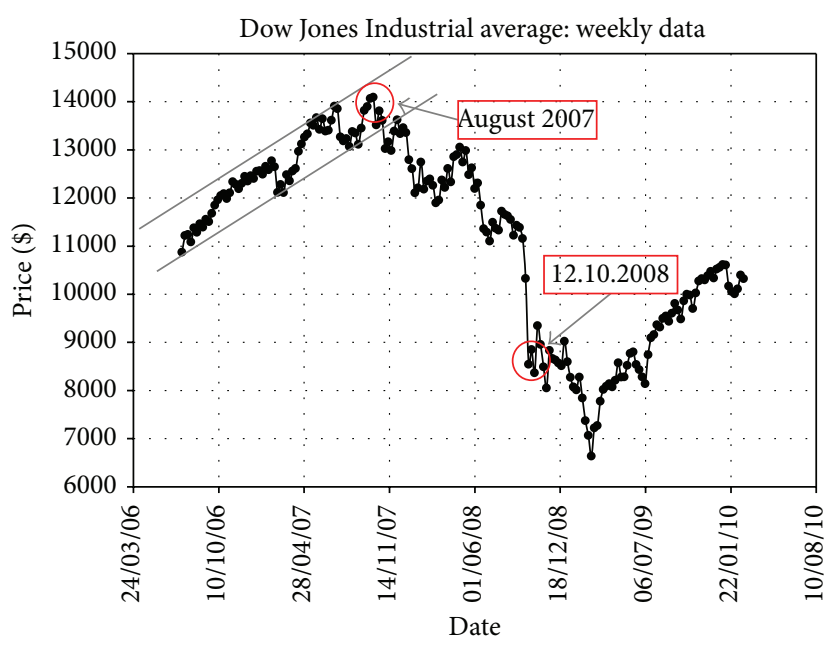

Figure 2: DJI weekly time series. Two stages of crisis—a failure of bank liquidity in August 2007 and the collapse of key mortgage agencies in September 2008.

chosen for a correct indication of the bifurcation: (a) $\dot{v}_{i}(t)>0$ and ${ }_{\Delta} \varepsilon_{i}\left(t_{i}\right) \prec 0$ or (b) $\dot{v}_{i}(t) \prec 0$ and ${ }_{\Delta} \varepsilon_{i}\left(t_{i}\right)>0$. Let us compose a time dependence of a product $\mathrm{Bif}_{i}={ }_{\Delta} \varepsilon_{i} \cdot \dot{v}_{i}$, where acceleration is expressed by

$$
v_{i}=\frac{x_{i}-x_{i-1}}{{ }_{\Delta} t_{i}}, \quad \dot{v}_{i}=\frac{v_{i}-v_{i-1}}{{ }_{\Delta} t_{i}}, \quad i=\overline{1, N} .
$$

An appearance of a disruption, then, corresponds to the transition $\mathrm{Bif}_{i}=0 \Rightarrow \mathrm{Bif}_{i} \prec 0$. A chart of a normalized bifurcation indicator is represented in Figure 3. We can note that a minimal value corresponds to the largest index fall of September-October 2008: the failure of greatest American mortgage agencies and a mortgage crisis in USA. Let us mark the date points of a critical bifurcation parameter $\mathrm{Bif}_{i}<0$. They are denoted by red points on date axis in Figure 4 . We have marked four clusters of critical points, corresponding to bifurcations: $14.10 .07-24.02 .2008,04.05 .2008-22.06 .2008$, 17.08.2008-05.10.2008, and 11.01.2000-15.02.2009. First cluster defines a delay between a bank crisis and a market reaction; we may observe inertial properties of a market system. However, $R$-analysis shows preliminary signals before a trend channel breakthrough. A third cluster corresponds to the mortgage crisis and a minimal Bif ${ }_{i}$ of Figure 3.

A second approach that should be considered is a diffusive Markovian analysis, represented above. The transport factor approximation has been calculated on the basis of relations (22). Its normalized values are displayed in Figure 5.

Highest amplitude of $D_{i}$ fluctuation again corresponds to October 2008, the mortgage crisis. Each intersection of a date axis indicates a new Markovian bifurcation. Points of intersections have been defined through the following condition:

$$
{ }_{\Delta} D\left(x_{i-1}, T\right) \cdot{ }_{\Delta} D\left(x_{i}, T\right) \prec 0 .
$$

This relation allows determining of derivative sign change: ${ }_{\triangle} D\left(x_{i-1}, T\right)>0 \Rightarrow{ }_{\Delta} D\left(x_{i}, T\right) \prec 0,{ }_{\Delta} D\left(x_{i-1}, T\right) \prec 0 \Rightarrow$ ${ }_{\Delta} D\left(x_{i}, T\right)>0$.

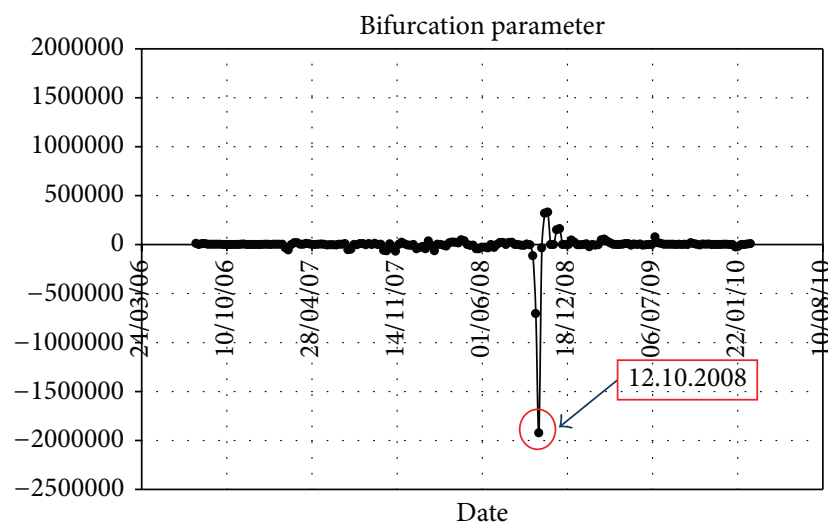

FIGURE 3: Bifurcation parameter time series: 16.07.2006-21.02.2010.

In Figure 6, principal clusters are marked on the OX axis as it has been done in Figure 4. It is significant to emphasize that an $R$-analysis has a wider area of application since it is not limited by Markovian processes and may be applied to processes with long memory, like inertial trends.

In Figure 6, areas of disruption, defined by both $R$ analysis and $D$-analysis, are marked by blue rectangles. Thus, a diffusive analysis has demonstrated $50 \%$ efficiency in relation to the $R$-description.

Let us designate a combination of $R$-analysis and $D$ analysis as $R / D$-analysis. This combined type of a description allows distinguishing of short-memory, Markovian stages of a market evolution and long-memory processes. Considered historical period of 2006-2010 allowed finding out of two short-memory periods-14.10.07-24.02.2008 and $04.05 .2008-22.06 .2008$ - and two long-memory stages17.08.2008-05.10.2008 and 11.01.2009-15.02.2009.

If we define a financial crisis as a systematic, inertial large scale failure of basic economic indexes, then, according to $R / D$-analysis, its beginning corresponds to October 2008 . 


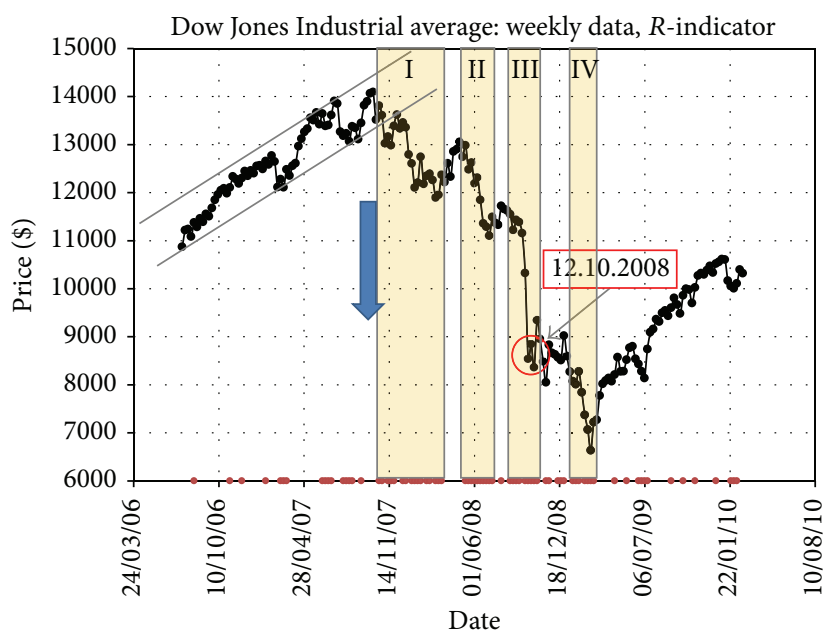

FIgURE 4: Clusters of a critical bifurcation: $R$-analysis.

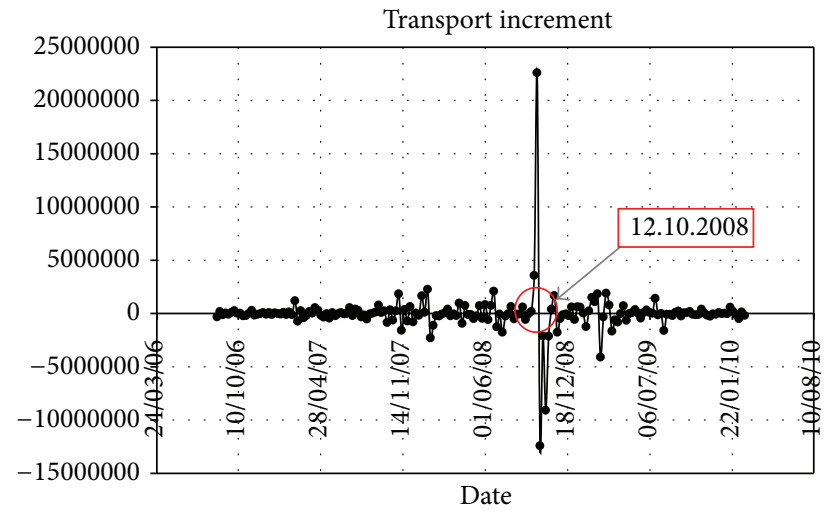

FIGURE 5: A transport increment time series: 16.07.2006-21.02.2010.

This is a date of propagation beginning through adjacent developed markets like markets of European Union.

\section{Conclusions}

In this paper, a combined transport analysis has been considered for the description of a precatastrophic stabilization. PS-effect has been stated as a fusion of stable and unstable parametric areas and phase cycles. A delay between a new cycle appearance and a macroscale excitation is defined by inertial properties of system domains-an indication of transport anomalies may help to forecast a catastrophe. We have shown that a classical monofractal analysis introduces artificial properties into physical process: nondifferentiability, Brownian nature, and linear memory measure. In this frame, a classical Hurst factor cannot be used for the indication of nonlinear precatastrophic stabilization.

A sense of a Hurst factor has been extended by the consideration of a phase diffusion law. This model characterizes a shift of a basic absorption band from a microscale to a macroscale range when a coherent motion and resonances appear. To indicate this effect, momentary phase diffusion

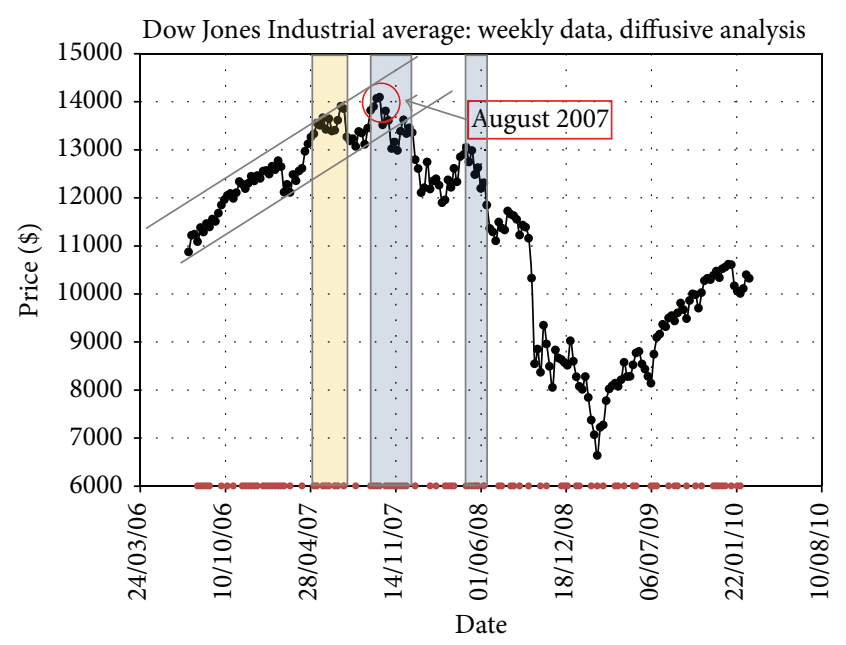

FIGURE 6: Clusters of a critical bifurcation: diffusive analysis.

has been introduced. It reaches a new minimum during a precatastrophic stabilization phase: ${ }_{\Delta} D\left(x_{i}, T\right)=0$. A disruption leads to a following increase of the momentary transport due to a large scale motion.

An efficiency of a diffusive analysis has been experimentally compared to the Reynolds stability model application. It has been shown that an $R$-analysis has a wider area of application since it is not limited by Markovian processes and may be applied to processes with long memory, like inertial trends. A combined diffusive and Reynolds analysis has been applied for a description of a time series of Dow Jones Industrial weekly prices during the world financial crisis of 2007-2009. Diffusive and Reynolds parameters showed extreme values in October 2008-the failure of greatest American mortgage agencies. This combined type of description has allowed distinguishing of short-memory Markovian stages and longmemory processes of a market evolution. The considered historical period of 2006-2010 allowed finding out of two short-memory periods. It was stated that a systematic large scale failure of a financial market began in October 2008 and started fading in February 2009.

\section{Conflict of Interests}

The author declares that there is no conflict of interests regarding the publication of this paper.

\section{References}

[1] H. Whitney, "On singularities of mappings of euclidean spaces. I. Mappings of the plane into the plane," Annals of Mathematics, vol. 62, no. 3, pp. 374-410, 1955.

[2] V. M. Zakaljukin, "Lagrangian and legendre singularities," Functional Analysis and Its Applications, vol. 10, no. 1, pp. 2636, 1976.

[3] Y. Kuznetsov, Elements of Applied Bifurcation Theory, vol. 112 of Applied Mathematical Sciences, 3rd edition, 2004.

[4] M. M. Dubovikov, N. V. Starchenko, and M. S. Dubovikov, "Dimension of the minimal cover and fractal analysis of time 
series," Physica A: Statistical Mechanics and Its Applications, vol. 339, no. 3-4, pp. 591-608, 2004.

[5] A. Neishtadt, "On stability loss delay for a periodic trajectory," Progress in Nonlinear Differential Equations, vol. 19, p. 253, 1996.

[6] S. Kamenshchikov, "Nonlinear Prigozhin theorem," Chaos and Complexity Letters, vol. 8, pp. 63-71, 2014.

[7] B. B. Mandelbrot and J. W. van Ness, "Fractional Brownian motions, fractional noises and applications," SIAM Review, vol. 10, pp. 422-437, 1968.

[8] E. E. Peters, Chaos and Order in the Capital Markets: A New View of Cycles, Prices, and Market Volatility, John Wiley \& Sons, 2nd edition, 1991.

[9] J. L. Lopez and J. G. Contreras, "Performance of multifractal detrended fluctuation analysis on short time series," Physical Review E, vol. 87, Article ID 022918, 2013.

[10] S. A. Kamenshchikov, "Clustering and uncertainty in perfect chaos systems," Journal of Chaos, vol. 2014, Article ID 292096, 6 pages, 2014.

[11] G. M. Zaslavsky and R. Z. Sagdeev, Introduction to Nonlinear Physics: From the Pendulum to Turbulence and Chaos, Nauka, Moscow, Russia, 1988.

[12] G. Soros, The New Paradigm for Financial Markets: The Credit Crisis of 2008 and What It Means, PublicAffairs, 2008. 


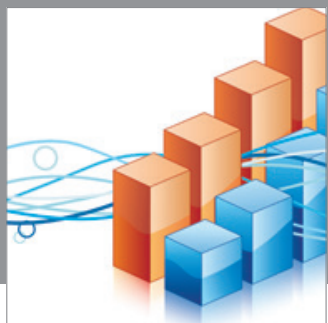

Advances in

Operations Research

mansans

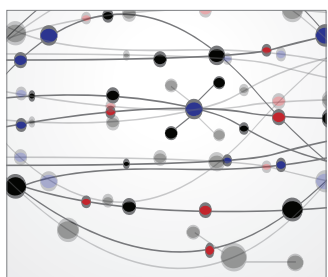

The Scientific World Journal
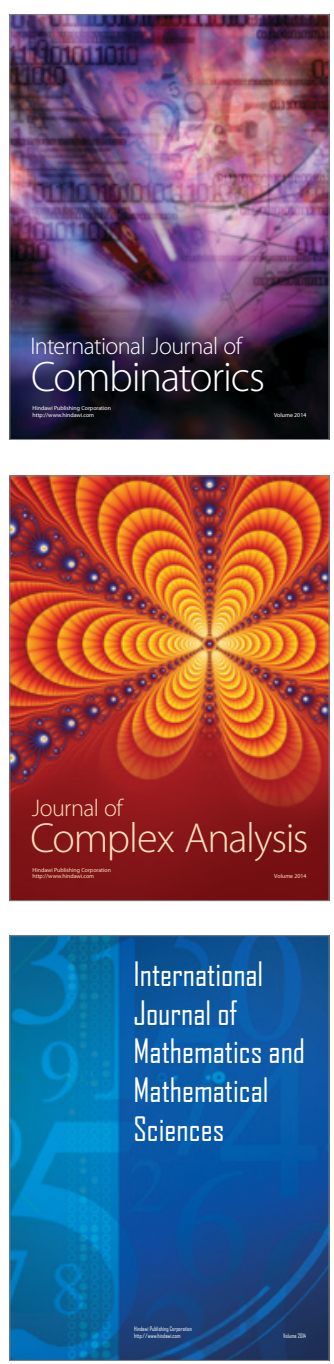
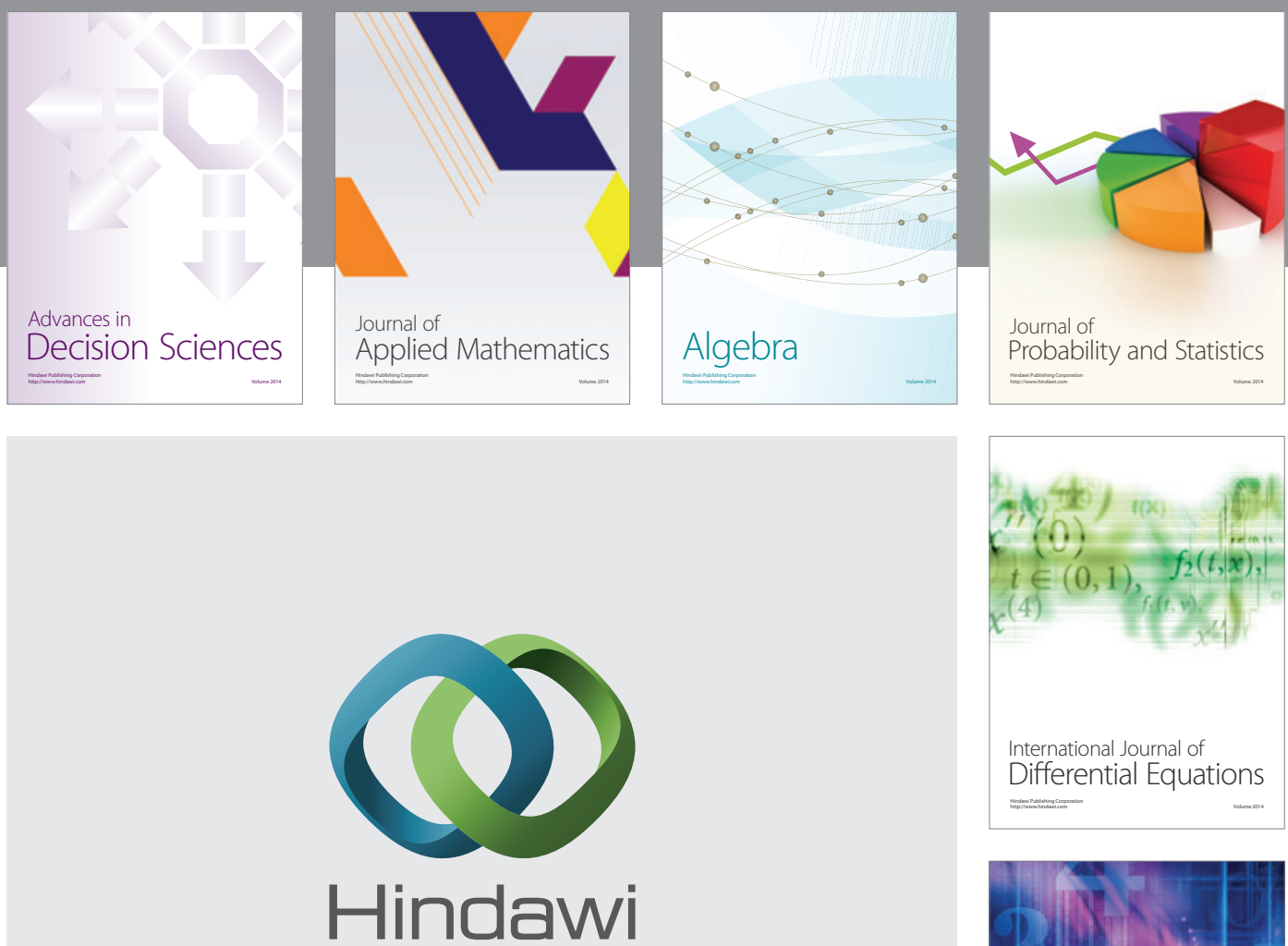

Submit your manuscripts at http://www.hindawi.com
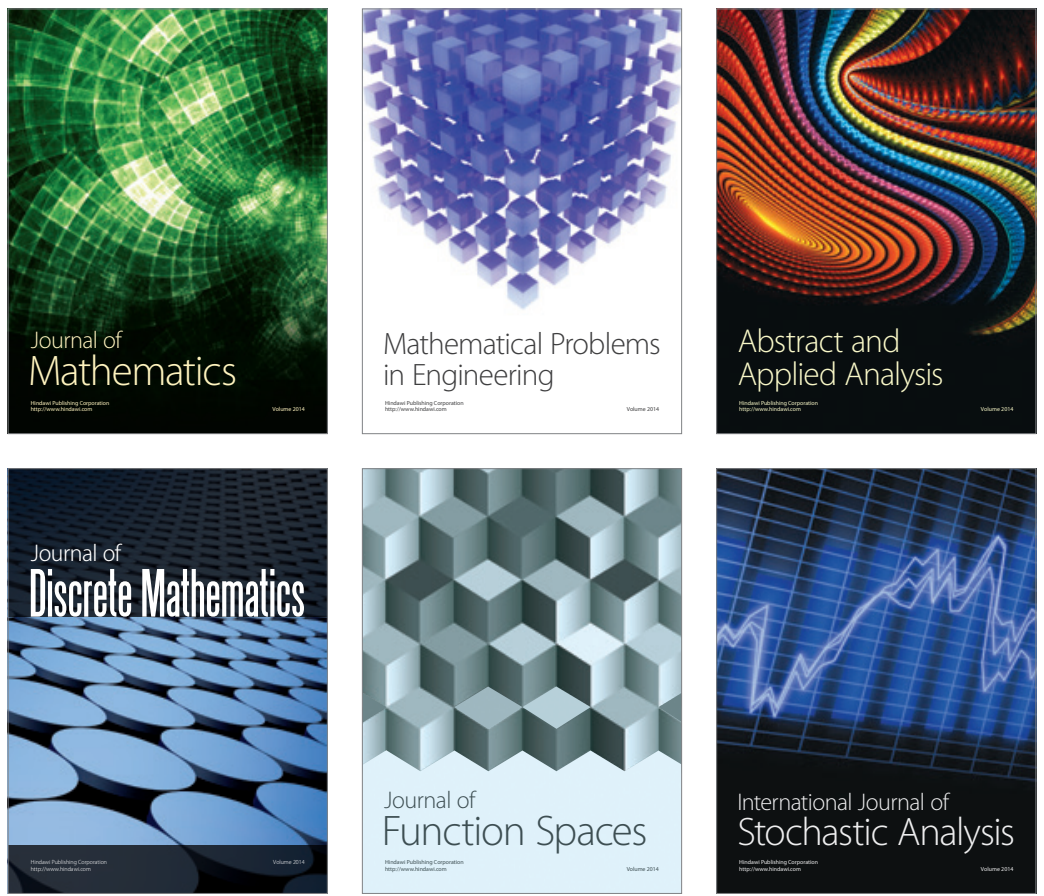

Journal of

Function Spaces

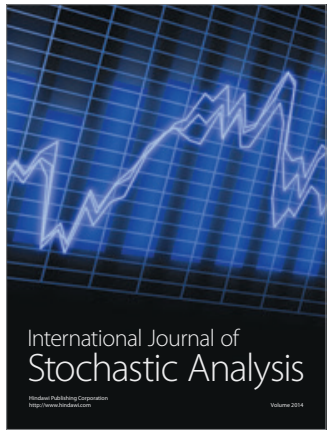

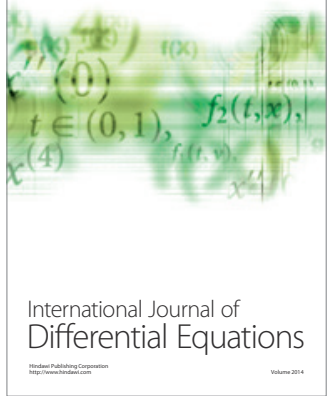
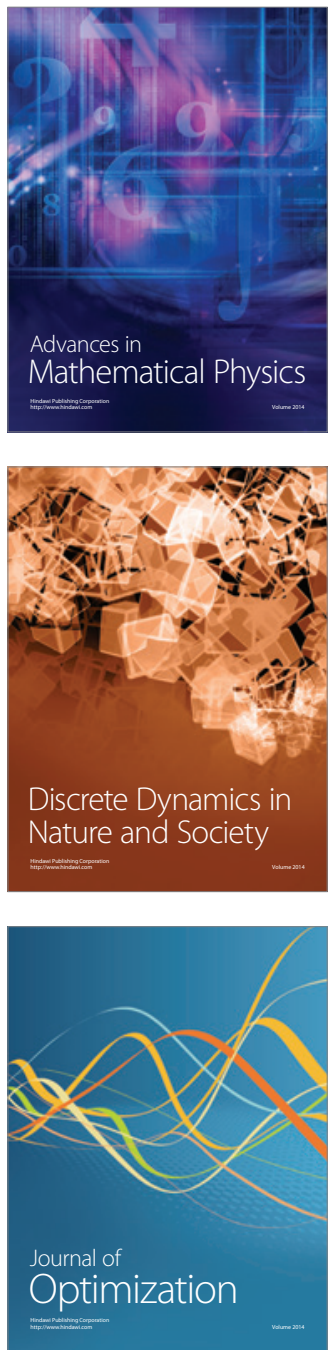